\title{
The Development and Implementation of a Cryogenic Pressure Sensitive Paint System in the National Transonic Facility
}

\author{
A. Neal Watkins, ${ }^{1}$ Bradley D. Leighty, ${ }^{2}$ and William E. Lipford ${ }^{3}$ \\ NASA Langley Research Center, Hampton, VA, 23681, USA \\ Donald M. Oglesby ${ }^{4}$ and Kyle Z. Goodman ${ }^{5}$ \\ ATK, Hampton, VA, 23681, USA \\ and \\ William K. Goad, ${ }^{6}$ Linda R. Goad, ${ }^{7}$ and Edward A. Massey ${ }^{8}$ \\ Jacobs Sverdrup, Hampton, VA, 23681, USA
}

\begin{abstract}
The Pressure Sensitive Paint (PSP) method was used to measure global surface pressures on a model at full-scale flight Reynolds numbers. In order to achieve these conditions, the test was carried out at the National Transonic Facility (NTF) operating under cryogenic conditions in a nitrogen environment. The upper surface of a wing on a full-span 0.027 scale commercial transport was painted with a porous PSP formulation and tested at $120 \mathrm{~K}$. Data was acquired at Mach 0.8 with a total pressure of $200 \mathrm{kPa}$, resulting in a Reynolds number of $65 \times 10^{6} / \mathrm{m}$. Oxygen, which is required for PSP operation, was injected using dry air so that the oxygen concentration in the flow was approximately $1535 \mathrm{ppm}$. Results show qualitative agreement with expected results. This preliminary test is the first time that PSP has been successfully deployed to measure global surface pressures at cryogenic condition in the NTF. This paper will describe the system as installed, the results obtained from the test, as well as proposed upgrades and future tests.
\end{abstract}

\section{Nomenclature}

PSP $\quad=$ Pressure Sensitive Paint

TSP $=$ Temperature Sensitive Paint

NTF $\quad=$ National Transonic Facility

UV $\quad=$ Ultraviolet

LED $\quad=$ Light Emitting Diode

$I \quad=$ Intensity of light emitted by PSP

$K_{S V} \quad=$ Stern-Volmer constant

$P \quad=$ Pressure

$A, B \quad=$ PSP calibration coefficients with arbitrary pressure reference

$\operatorname{Pt}(\mathrm{TfPP})=$ platinum meso-tetra(pentafluorophenyl) porphine

PTMSP $=$ polytrimethylsilylpropyne

\footnotetext{
${ }^{1}$ Aerospace Technologist, Advanced Sensing and Optical Measurement Branch, Mail Stop 493, AIAA Member.

${ }^{2}$ Engineering Technician, Advanced Sensing and Optical Measurement Branch, Mail Stop 493.

${ }^{3}$ Engineering Technician, Advanced Sensing and Optical Measurement Branch, Mail Stop 493.

${ }^{4}$ Research Chemist, NASA Langley Research Center, Mail Stop 493.

${ }^{5}$ Research Chemist, NASA Langley Research Center, Mail Stop 493.

${ }^{6}$ Instrumentation Technician, NASA Langley Research Center, Mail Stop 267.

${ }^{7}$ Instrumentation Technician, NASA Langley Research Center, Mail Stop 267.

${ }^{8}$ Instrumentation Technician, NASA Langley Research Center, Mail Stop 267.
} 


\section{Introduction}

$\mathrm{T}$ HE Pressure Sensitive Paint (PSP) technique was used to measure global surface pressures on a transport wing model at full-scale flight Reynolds numbers. This test was conducted at the National Transonic Facility (NTF) at NASA Langley Research Center in Hampton, Virginia, and full-scale flight Reynolds numbers were achieved at cryogenic conditions. Traditionally, surface measurements conducted at cryogenic conditions require the use of hardened instrumentation such as pressure taps. Instrumenting models with these is typically limited by the shape and thickness of the model as well as available space for the support instrumentation. Typically multiple models with and without pressure instrumentation are often required to perform full aerodynamic characterization.

PSP allows for the accurate determination of pressure and temperature distributions over an aerodynamic surface and is based on an emitted optical signal from a luminescent coating. As originally developed, ${ }^{1-3}$ this technique was primarily useful for mean pressure measurements at transonic and higher flows, but has since been adapted to lower speed flows ${ }^{4-6}$ as well as measurements of fluctuating pressures. ${ }^{7,8}$ A number of review articles cover the topic in detail. ${ }^{9-11}$ PSP measurements exploit the oxygen $\left(\mathrm{O}_{2}\right)$ sensitivity of luminescent probe molecules suspended in gaspermeable binder materials. In wind tunnel applications, the PSP is applied to the model by conventional paint spraying techniques. Light sources such as UV LED arrays are mounted external to the test section to illuminate the painted model and effect luminescence emission from the entrapped oxygen-sensitive molecules. For the majority of pressure paints, PSP emission occurs in the red or orange region of the visible spectrum $(\sim 580-650 \mathrm{~nm})$. The intensity is inversely proportional to the amount of oxygen present such, that brighter regions in the paint emission indicate lower concentration of oxygen (and thus lower pressure) relative to the darker regions. Scientific-grade CCD cameras with spectral band-pass filters to discriminate between the excitation (blue) and emission (orange) signals, capture the intensity image of the PSP-coated model surface, providing a means to recover global surface pressure distributions on test articles of interest. PSP measurement systems all employ a ratio of image pairs to compensate for intensity non-uniformity due to sources other than oxygen concentration, the most significant of which are paint application and illumination heterogeneity. In the conventional approach, PSP images acquired either prior to or immediately following tunnel operation (wind-off) are ratioed with images acquired at each tunnel condition (wind-on). A companion technique, TSP, uses probe molecules whose brightness varies with temperature, but which are insensitive to oxygen concentration.

If the test surface under study is immersed in an atmosphere containing $\mathrm{O}_{2}$ (e.g. air), the recovered luminescence intensity can be described by the Stern-Volmer relationship ${ }^{12}$

$$
\frac{\boldsymbol{I}_{0}}{\boldsymbol{I}}=1+\boldsymbol{K}_{S V} \boldsymbol{P}_{\boldsymbol{O}_{2}}
$$

where $I_{0}$ is the luminescence intensity in the absence of $\mathrm{O}_{2}$ (i.e. vacuum), $I$ is the luminescence intensity at some partial pressure of oxygen $P_{O 2}$, and $K_{S V}$ is the Stern-Volmer constant. The value of $K_{S V}$ depends on the properties of both the luminescent molecules and the binder, and is generally temperature dependent. Since it is a practical impossibility to measure $I_{0}$ in a wind tunnel application, a modified form of the Stern-Volmer equation is typically used. This form replaces the vacuum calibration $\left(I_{0}\right)$ with a reference standard

$$
\frac{I_{R E F}}{I}=A+B \frac{P}{P_{R E F}}
$$

where $I_{R E F}$ is the recovered luminescence intensity at a reference pressure, $P_{R E F}$. $A$ and $B$ are temperature dependent constants for a given PSP formulation and are usually determined before hand using laboratory calibration procedures. The calibration and interpretation of TSP data follows a similar procedure, using the TSP luminescence intensity recovered at a reference temperature.

PSP measurements are difficult to make under cryogenic conditions for two reasons. First, the test gas is typically nitrogen, refrigerant, or some other medium which typically contains little or no oxygen. Second, the diffusion of oxygen into the paint binder is highly temperature dependent, and at low temperatures, is practically nonexistent. As such, it is not surprising that initial cryogenic testing with luminescent paints used TSP. ${ }^{13}$ Indeed; probe molecules can be found which respond strongly to temperature even under cryogenic conditions.

For PSP, researchers developed two distinct methods to overcome the challenges caused by the low diffusion of oxygen into the paint binder. Initial cryogenic PSP tests were conducted using anodized aluminum. ${ }^{14}$ In these tests, the model was constructed from aluminum and anodized to coat the surface with a monolayer of aluminum oxide. 

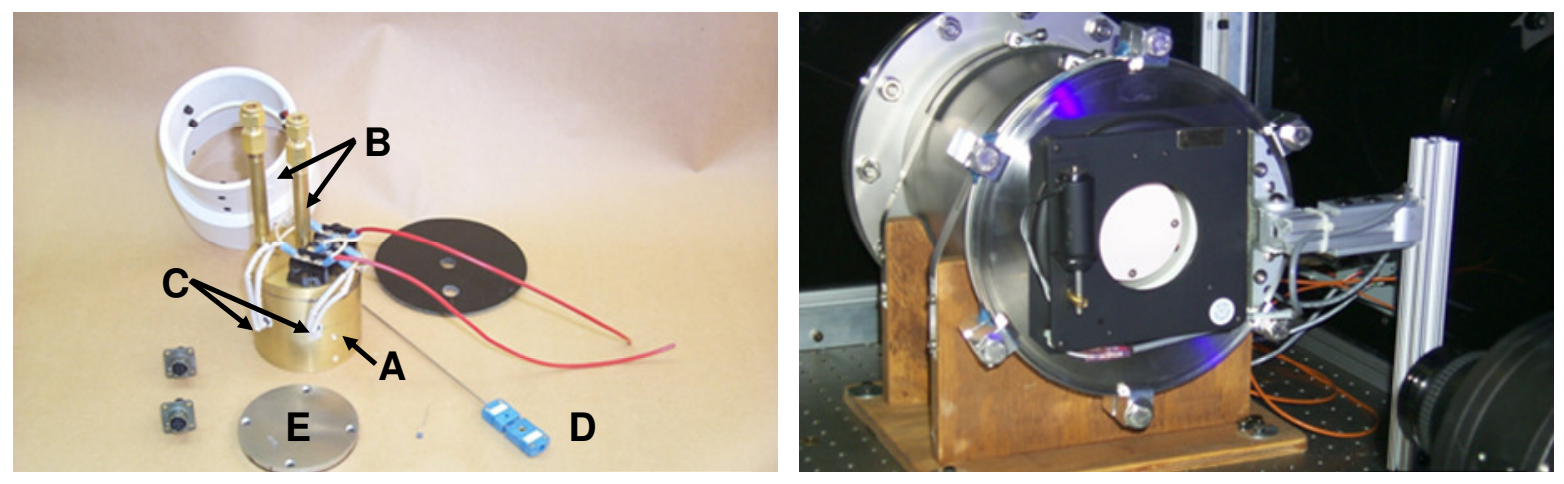

Figure 1. Calibration chamber for cryogenic PSP and TSP formulations. The left picture depicts the interior of the chamber including the brass block (A), the inlet and outlet for liquid nitrogen flow (B), the resistive heaters $(\mathrm{C})$, the thermocouple for temperature monitoring and control (D), and an unpainted coupon (E). The right picture shows the complete calibration chamber with a coupon and the external shutter open.

Then the model was coated with an oxygen luminophore that chemically bonds with the aluminum oxide. This produces a coating that is essentially a few molecules thick, thus alleviating the issues with paint binders and oxygen diffusion. The disadvantages to this technique are that the anodizing requirement makes it difficult to use with large and complex models, and it cannot be used with the stainless steel models that are typically employed in large-scale cryogenic testing. For this testing, a PSP formulation consisting of a binder that has a very large diffusion rate and can be applied with standard airbrush techniques and capable of adhering to many different types of surfaces was developed. ${ }^{15,16}$ These formulations have been used successfully at the NASA Langley 0.3-Meter Transonic Cryogenic Tunnel ${ }^{15}$ as well as other facilities ${ }^{16}$ A variation of the PSP technique has been performed at the NTF with this formulation to visualize surface flow patterns on a blended wing body model, ${ }^{17}$ and preliminary testing of cryogenic PSP at the European Transonic Windtunnel has also been reported. ${ }^{18}$

\section{Experimental}

\section{A. PSP Formulation}

The PSP formulation used in the current study was made by dissolving an oxygen sensitive luminophore, $\mathrm{Pt}(\mathrm{TfPP})$, in an oxygen-permeable binder. The oxygen-permeable binder used was PTMSP, ${ }^{15,16,19}$ chosen because it is a glassy polymer with a large free volume and having a low diffusion barrier to oxygen. This leads to high oxygen permeability even at cryogenic conditions. Calibration of the paint was performed using a specially designed calibration cell. The calibration cell, shown in Figure 1, consists of a brass block (A). Temperature control of the block is accomplished by flowing liquid nitrogen into the block through milled channels internal to the block (B) and heat is applied via resistive heaters (C) imbedded in the block. A thermocouple (D) is also imbedded into the block, which serves as the sensor input to a proportional-integral-derivative (PID) controller capable of regulating both the amount of liquid nitrogen flowing to the cell (via a hardened solenoid valve) and the amount of heat applied. A painted coupon (E) is bolted to the front of the block and thermal contact is ensured using a siliconfree thermal compound. Pressure is controlled by sealing he block in a chamber equipped with a quartz window. An external shutter is affixed to the window to reduce photodegradation of the paint. The chamber is also placed inside a dark enclosure to minimize background illumination. The cell is capable of operating from vacuum to room atmosphere and to temperatures as low as $77 \mathrm{~K}$ (boiling point of liquid nitrogen). An aluminum or steel coupon was coated with the PSP formulation, placed in the cell, and illuminated using a $400 \mathrm{~nm}$ LED array. Luminescent images were then acquired using a CCD camera equipped with a band-pass filter to block any excitation light present. Oxygen-nitrogen gas mixtures were used to mimic the expected condition in the NTF. A typical PSP response curve to increasing amounts of a 3000 ppm oxygen-nitrogen mixture is shown in Figure 2. 


\section{B. Facility}

The NTF is one of the world's leading facilities for providing high quality flight Reynolds number aeronautical data and has been operational since 1982. ${ }^{*}$ The tunnel has a test section of 2.5 meters x 2.5 meters and is capable of operating at speeds from subsonic $(M=0.1)$ to transonic $(M=1.1)$ with Reynolds numbers from $13.1 \times 10^{6} / \mathrm{m}$ to $476 \times 10^{6} / \mathrm{m}$ at transonic Mach numbers. The tunnel is capable of operating in either air mode at elevated pressure and temperature or at cryogenic conditions (to $117 \mathrm{~K}$ ) by injecting liquid nitrogen into the tunnel circuit. In the cryogenic operating mode, the NTF is capable of providing full-scale flight Reynolds numbers without an increase in model size. Several optical diagnostic techniques are available in the NTF, including Video Fluorescent Minituft Flow Visualization, Sharp Focusing Schlieren Flow Visualization, Video Model Deformation, and a newly designed and installed PSP/TSP system.

The major challenge to conducting PSP tests at cryogenic conditions is the introduction of a suitable amount of oxygen to facilitate paint sensitivity. For the preliminary testing, dry air (dew point $<210 \mathrm{~K}$ ) from a centralized compressor station was chosen as opposed to liquid oxygen to minimize safety risks. However, the selection of an injection point in the

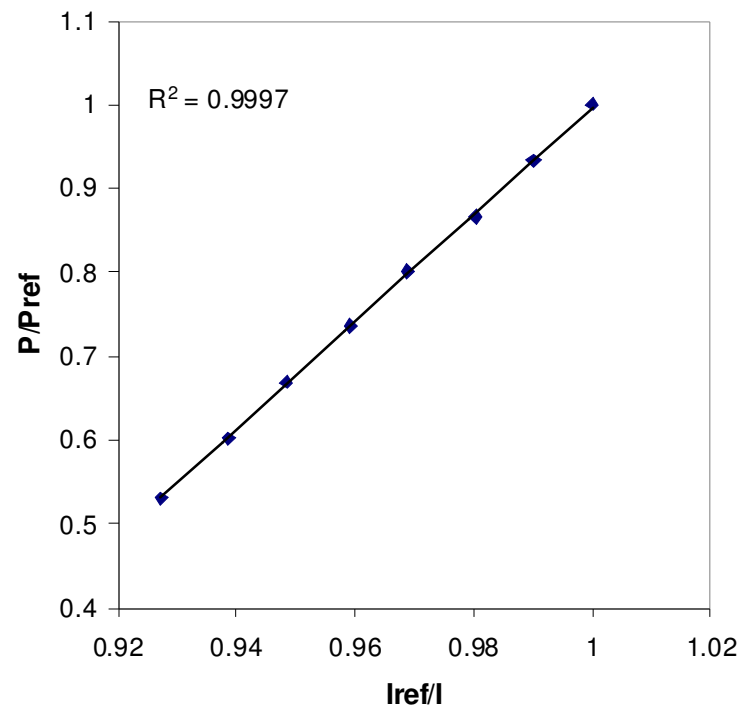

Figure 2. Typical PSP response curve to increasing gas pressure. The gas used was $3000 \mathrm{ppm}$ oxygen in nitrogen and the data was collected at approximately $137 \mathrm{~K}$. tunnel that will allow rapid entrainment and circulation of the oxygen in the flow is critical. Figure 3 shows the layout of the NTF with potential injection stations labeled at A, B, and C. The station with the longest transit path before passing the model is station A at the trailing edge of the model support sting. However, this would require extensive modification of the support sting, possibly causing interference with tunnel operation. In addition, a long term goal of this project would be to use the station as a possible source to inject "particles" in the stream for flow visualization. At point A, the long transit path would also allow for potential particle settling out of the flow in the low speed sections and depositing on the structures in the flow path (especially in the corner turning vanes). Station $\mathrm{C}$ (in the rapid diffuser just before the cooling coil) would result in essentially zero chance of particles plating out, but would also have the lowest transit time (thus less mixing) and would affect the flow in the tunnel to the greatest extent. Station B was ultimately chosen as it was deemed to have the smallest effect on flow quality (as it is in the turbulent region of the tunnel) while still giving a uniform distribution again due to the higher turbulence in the region.

Air injection in this test was accomplished using a valve to regulate the air flowing into the tunnel. Numerical studies of the flow at the NTF estimated that approximately $0.9 \mathrm{~kg} / \mathrm{s}$ dry air would be required to achieve an oxygen concentration of $1000 \mathrm{ppm}$ in the flow at a tunnel temperature of $117 \mathrm{~K}$ and pressure of $275 \mathrm{kP}$. Little if any tunnel heating effects were observed using these magnitude flow rates, as determined by the negligible temperature changes noted in the flow. This could be expected as liquid nitrogen is introduced to the flow under these conditions at approximately $130 \mathrm{~kg} / \mathrm{s}$. Oxygen monitors (described below) were interfaced into the tunnel and monitored to determine when and how much air would be introduced. The major concern with using air as the oxygen source is the potential introduction of water into the tunnel circuit. At cryogenic conditions, this could produce frost in the tunnel or on the model which can have dramatic effects on the measured aerodynamic characteristics. No evidence of frost was detected on the model for this test, but this continues to be a major concern, and alternate strategies such as the use of additional dryers or liquid oxygen (instead of air) are being explored to mitigate this contingency.

${ }^{*}$ For additional information, please visit http://wte.larc.nasa.gov/facilities/aerodynamics/national.cfm 


\section{Model}

The model is a full-span 0.027 scale model of a commercial transport. The design of the model was a joint effort between NASA and Microcraft Incorporated, and was designed so that it would be suitable for testing at the high pressure, cryogenic conditions in the NTF. The entire model has a finish of approximately $0.2 \mu \mathrm{m}$, and it is mainly composed of 13-8 stainless steel, with the wings and flow-through nacelles composed of Vascomax 250 and Vascomax 200, respectively. The model can also be outfitted with horizontal tails, flap support fairings, and varying wingtip designs. It can be supported in the test section in varying arrangements, although the more popular method is via an upper swept

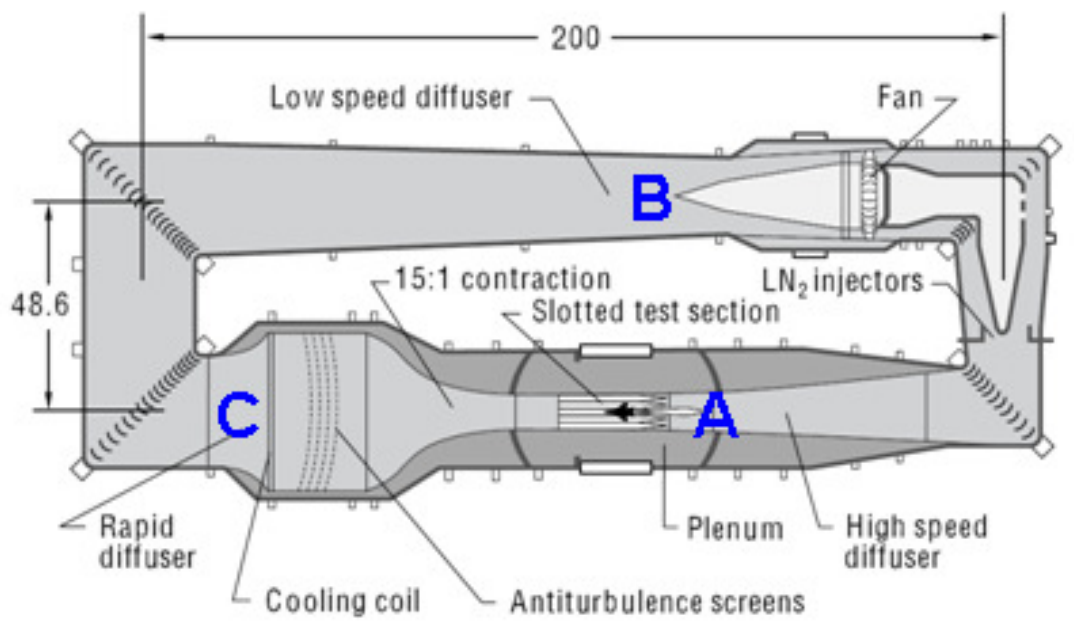

Figure 3. NTF tunnel circuit arrangement depicting possible injection points. point $A$ is at the trailing edge of the model support system; point $B$ is in the turbulent region near the low speed diffuser; point $C$ is in the rapid diffuser just before the cooling coil.

strut that enters the model in the upper empennage where a vertical tail would normally reside. A bulb-type seal is normally used in this arrangement to prevent flow from entering the fuselage. The model has a wingspan of approximately $1.5 \mathrm{~m}$ and it weighs approximately $136 \mathrm{~kg}$.

Previous cryogenic PSP $^{15}$ and TSP $^{20}$ tests have shown that highly polished stainless steel models must first be painted with a basecoat before the PSP will adhere to the model. For this test, SpectraPrime (Sherwin Williams) was chosen as the basecoat. Previous tests have shown this paint to be very robust to cryogenic conditions. The SpectraPrime basecoat must be cured and was accomplished by exposing it to heat lamps in the test section for approximately 3 hours. After curing, the basecoat layer was sanded with 1500-2000 grit paper, which was needed to promote adhesion of the PSP layer. Finally, the PTMSP PSP was applied to the model, with a final thickness (basecoat and PSP layer) of approximately $40 \pm 8 \mu \mathrm{m}$ (as measured using an electronic thickness gauge), and the roughness was approximately $0.4 \pm 0.08 \mu \mathrm{m}$ (as measured using a stylus-based surface roughness tester), approximating the original finish of the model.

\section{Instrumentation}

Illumination: Illumination was achieved using several custom designed LED-based arrays capable of operating at either room temperature (for NTF air model operation) or at cryogenic conditions. The arrays (shown in Figure 4) consisted of 80 individual LED elements arranged on a $12.7 \mathrm{~cm}$ diameter $4.5 \mathrm{~mm}$ thick aluminum substrate. The aluminum substrate is also equipped with an RTD sensor to monitor temperature as well as resistive heaters on the back for cryogenic operation. The LED arrays are operated using a $1 \mathrm{~kW}$ switching power supply and can be remotely operated using standard TTL (transistor-transistor logic) pulses. The LED array produces light centered at $400 \mathrm{~nm}(\sim 20 \mathrm{~nm}$ full width at half maximum) at greater than $80 \mathrm{~W}$ under nominal forward current conditions. This can also be manually adjusted as needed by controlling the current applied to each array. A total of eight of these arrays were positioned in available port holes to illuminate the upper surface of the model. 
Image acquisition: Images were acquired from cameras placed directly over the model as well as cameras placed in the sides of the tunnel positioned so as to view the top of the model obliquely. The ports in the ceiling and floor are not big enough for an environmental chamber, so these cameras viewing the model from the top were specially designed to withstand the elevated pressures and cryogenic temperatures that are experienced in the plenum. The cameras are slow-scan back-illuminated scientific grade CCDs with 16-bit digitization and $1024 \times 1024$ pixel resolution. Because of the extreme environment, no shutters are employed on these cameras and all exposure is controlled by pulsing the LED arrays.

For viewing from the side wall, the area is of suitable size to allow the camera to be placed in an environmental chamber supplying sufficient heat to operate. This camera is front-illuminated with microlenses on each pixel to improve efficiency. The camera employs 14-bit digitization and 1600x1200 pixel resolution. Both camera systems were interfaced to the computer using custom designed armored fiber optic cables capable of operating at cryogenic temperatures.

Oxygen monitoring: To facilitate calibration of the paint, the oxygen concentration in the flow must be measured accurately. This was done by interfacing an oxygen monitoring system into the tunnel control loop. Two oxygen monitors were attached to outlets of pressure ports, one from a static port in the floor and one from a dynamic port in a small pressure rake. Both were mounted approximately $4.5 \mathrm{~m}$ forward of the model. The oxygen sensor system employed a zirconium oxide sensor with a time response of less than $10 \mathrm{~s}$. The system was also equipped with an in situ calibration option allowing it to maintain linearity and repeatability of less than $2 \%$ or reading or $0.5 \mathrm{ppm} \mathrm{O}_{2}$ absolute. The unit is controlled by a personal computer via RS-232 protocol.

\section{E. Data Acquisition}

Data acquisition was performed using a modification of the typical procedure described above. Introduction of dry air into the tunnel was first performed using a valve and the oxygen concentration was monitored until it stabilized. Then wind-on images were acquired at various angles of attack with a wind speed of Mach 0.8 . The oxygen concentration was continuously monitored and when it began to decrease significantly, more air was introduced to re-establish the baseline value. Reference (or "wind-off") images were acquired immediately following an alpha sweep. Ideally, the tunnel would have been pumped completely free of oxygen and the tunnel flow ceased to acquire the wind-off image. However, the cost of this procedure, which would have taken several minutes and tons of nitrogen without data acquisition, precluded this. Therefore, the reference images were taken using the same level of oxygen in the flow. To ensure adequate mixing of oxygen in the flow for these reference images, the model was kept at a very low speed ( 0.08 Mach), causing as much as $1 \%$ error in the pressure measurements. For these tests, the measured oxygen concentration was maintained at $1535 \pm 35$ ppm for both the wind-on and the reference images. Prior to testing, background images and flat-field images were acquired and used as corrections for camera sensitivity and background light contamination. 


\section{Results and Discussion}

\section{A. PSP Test Results}

The initial focus of this work was to finalize the installation of the PSP/TSP system in the NTF as well as demonstrate the system in the relevant environment. This included interfacing the oxygen monitoring system into the measurement system, as well as determining the optimum procedures for introducing the required oxygen levels into the flow stream. For this test, the tunnel conditions were held constant at Mach 0.8 and $120 \mathrm{~K}$, with a total pressure of approximately $200 \mathrm{kPa}$, resulting in a Reynolds number of approximately $65 \times 10^{6} / \mathrm{m}$. During a run, the model was swept through angles of attack from -3 degrees to 2 degrees and PSP images were taken at 1 degree intervals.

Figure 5 shows reduced pressure data at various angles of attack acquired from both the ceiling camera and the camera in the side wall. The camera from the side wall cannot image the entire wing as the fuselage is obstructing its view. In Figure 5, a box is drawn around the ceiling camera image to depict the approximate viewing area of the side wall mounted camera. There is good qualitative and quantitative agreement between both camera views. The unpainted rows seen in the images are rows of pressure taps which were left unpainted for this test. As this was a demonstration test, the wing was left uninstrumented, thus all calibrations were made using the $a$ priori calibration

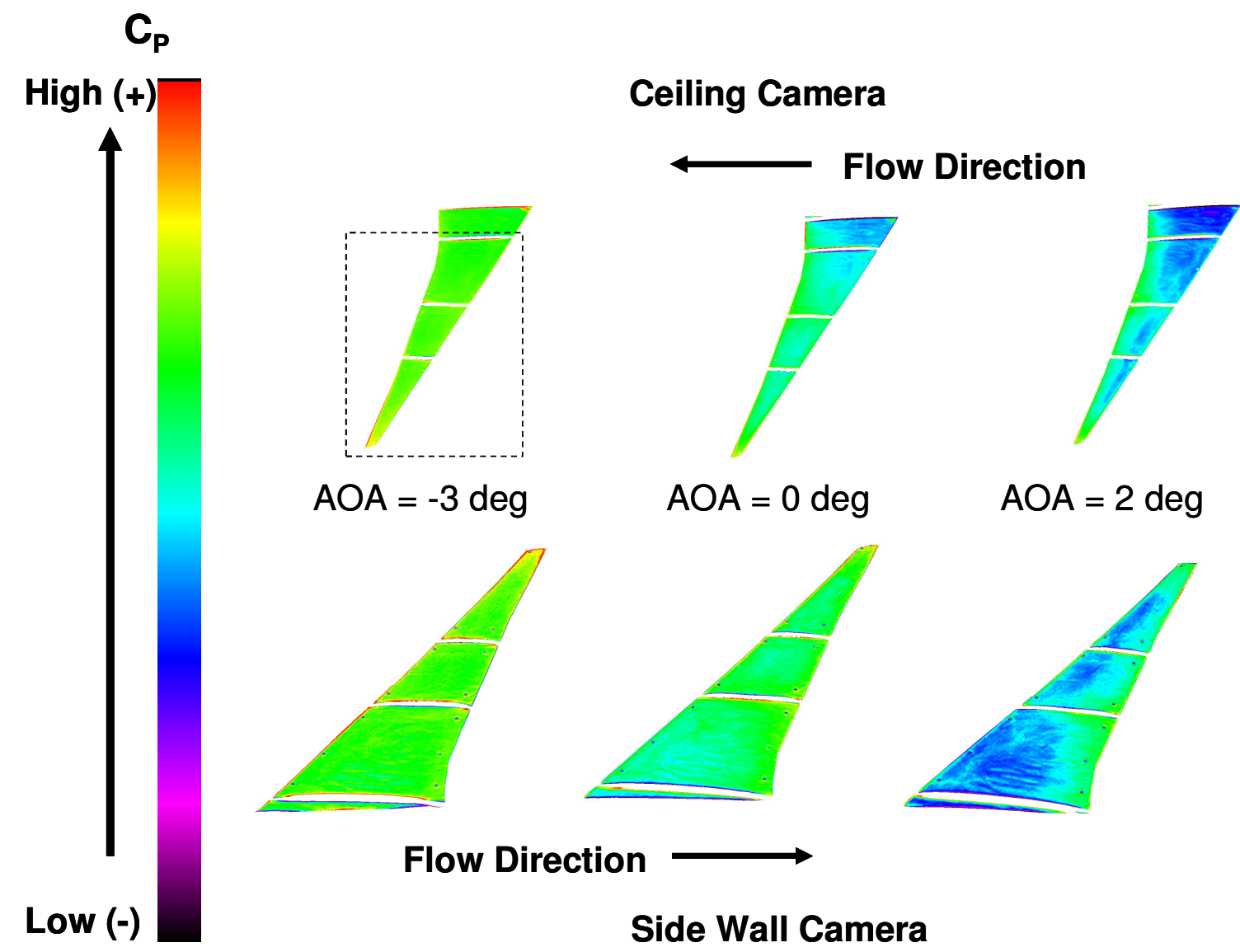

Figure 5. Representative PSP images acquired from the ceiling mounted camera (top) and the side wall mounted camera (bottom). The box drawn around the ceiling camera image shows the approximate viewing area of the side wall camera.

American Institute of Aeronautics and Astronautics 

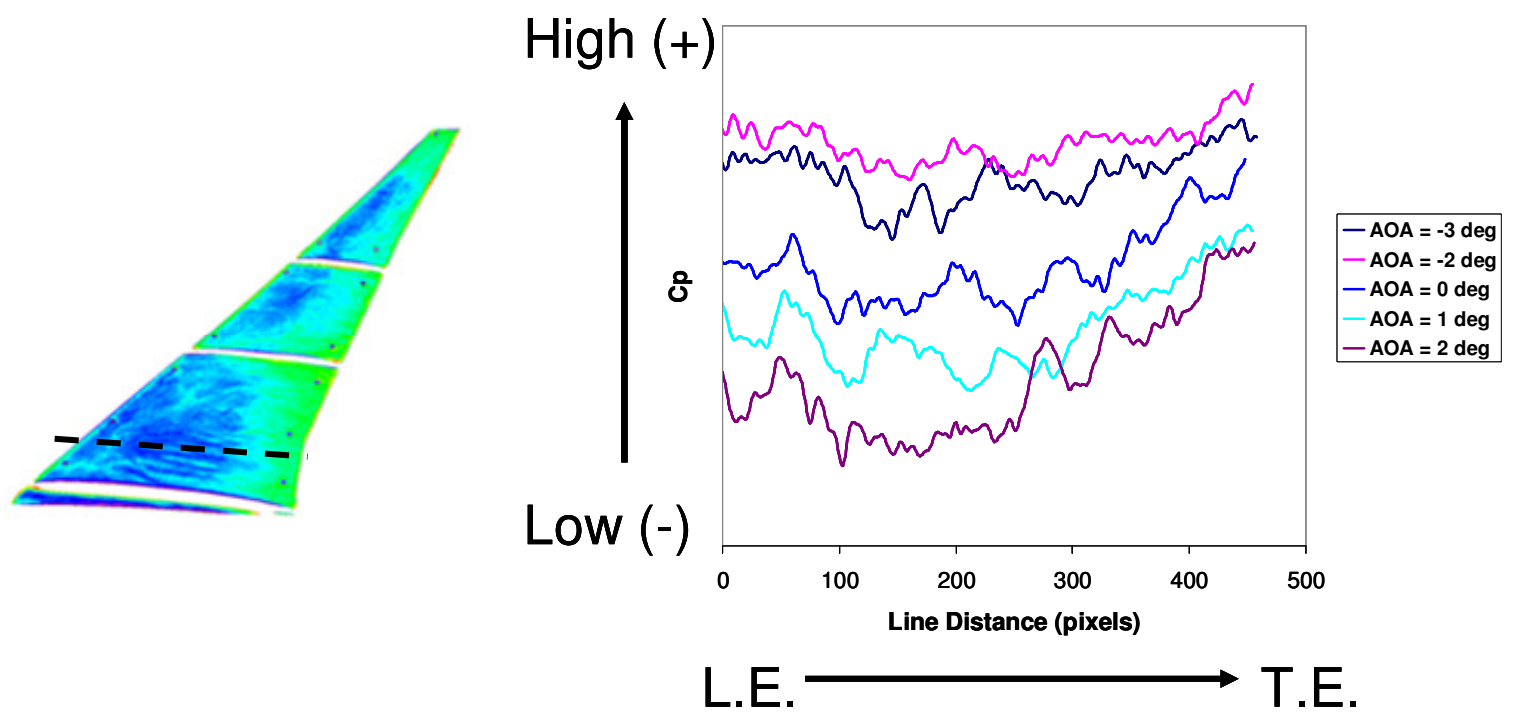

Figure 6. Representative slice from the images collected with the side mounted camera. The approximate location of the cut is shown in the image as the dotted line. This line was placed in the same physical location of the wing at each angle. The edges of the model are marked as L.E. (leading edge) and T.E. (trailing edge).

determined in the laboratory. However, qualitative behavior of the pressure on the wing seems reasonable as there is a clear lower pressure region (negative $\mathrm{Cp}$ ) on the leading edge with increasing pressure moving aft of the wing. The low pressure region on the leading edge also becomes much more pronounced as the model is put into a higher angle of attack, indicating acceleration of the flow. A cut of this data at approximately the same physical location on the wing at each angle of attack also shows this trend, as seen in Figure 6. Future tests will focus on validating the PSP results with an instrumented model, which will also allow the use of the in situ calibration technique for PSP. This will also be able to account for possible oxygen concentration gradients in the flow which may be occurring that are not measured at the oxygen monitoring station. In the current configuration, there is no other way to adequately account for this possibility. Additional testing also needs to be considered to determine if there is an effect on the flow characteristics around the model due to the presence of the paint layer. Previous testing at other facilities ${ }^{21}$ show this is unlikely due to the measured paint thickness and roughness, but this has not been confirmed at this time in the NTF.

\section{B. Proposed Improvements}

While the test demonstrated the effectiveness of PSP for measuring global surface pressures in full flight Reynolds number testing, several improvements have been indentified and are currently being implemented for future operation. While the current LED-based arrays for illumination have done very well, additional arrays have been acquired for future tests requiring illumination from more than one angle (for example, top and bottom simultaneously). Additionally, different color LED-based arrays are being investigated to ease the issues concerning surveillance of the model and test section during operation.

Newer cameras have also been identified and acquired for imaging from the ceiling. While the current cameras have been hardened specifically for the cryogenic operating environment of the NTF, their operating lifetime is approaching an end. For this test, one of the ceiling cameras became inoperable at the cryogenic conditions (probably due to a failure in its thermoelectric heater). In addition, since neither camera in the ceiling is equipped with a shutter, frame transfer artifacts can appear in the image especially if the CCD chip is not adequately cleaned (electronically) before image acquisition. The newer cameras are physically smaller than the existing cameras, allowing for some environmental conditioning to be added, yet have a higher resolution (4000x2672 pixels vs. $1024 \times 1024$ pixels) and transfer rate than the current cameras. Additionally, they are equipped with an electronic shutter similar to the cameras used for side viewing.

American Institute of Aeronautics and Astronautics 092407 
Another area of improvement is in better control of the oxygen concentration in the flow. The current method simply uses a valve that is manually controlled to introduce the required flow rate of dry air. This gave a small variability in the oxygen concentration (approximately $35 \mathrm{ppm}$ ), though still resulting in an error in the measured pressure of $\sim 3 \%$. Ideally, the valve should be replaced with one whose control range is larger and controlled by a flow controller that is interfaced into the oxygen monitoring system with a goal of having the variability in oxygen concentration approaching $5 \mathrm{ppm}$ or less. This should decrease the measurement error due to oxygen variability to less than $1 \%$.

\section{Conclusions}

The results from a proof of concept test using PSP to measure surface pressure distributions on a scale model at cryogenic conditions have been presented. These tests were conducted at full scale Reynolds number conditions at the NTF and show that the PSP technique is viable under these extreme conditions if suitable choice in paint and data acquisition techniques is chosen. The pressure contours measured on the surface agree qualitatively with expected results. Quantitative validation of the results was not possible because the pressure taps were not employed in this test. Thus several indeterminate errors are present, causing an unknown offset of the data.

Several modifications and improvements in the instrumentation and tunnel operation have been identified and are currently being implemented. These include the acquisition of more LED-based arrays to illuminate models from multiple angles and the identification and installation of new camera systems. In addition, a more advanced mechanism to control the oxygen concentration in the flow stream will be needed to minimize possible concentration variation with time that will result in an indeterminate error. Finally, additional tests using an instrumented model have been identified as a means to validate the technique as well as determine if the PSP coating is causing a measurable effect on aerodynamic characteristics. Regardless, this test has been a success and demonstrates the viability of using the PSP technique to optically determine surface pressures at larger scale cryogenic facilities.

\section{Acknowledgments}

The authors would like to thank Scott Goodliff and the rest of the personnel at the National Transonic Facility for their assistance during testing. This work was funded by NASA through the Subsonic Fixed Wing portion of the Fundamental Aeronautics Program.

\section{References}

\footnotetext{
${ }^{1}$ Kavandi, J., Callis, J., Gouterman, M., Khalil, G., Wright, D., Green, E., Burns, D., and McLachlan, B., "Luminescence Barometry in Wind Tunnels," Review of Scientific Instruments, Vol. 61, No. 11, 1990, pp. 3340-3347.

${ }^{2}$ Morris, M.J., Benne, M.E., Crites, R.C., and Donovan, J.F., "Aerodynamic Measurements Based in Photoluminescence," AIAA Paper 93-0175, 31 ${ }^{\text {st }}$ Aerospace Sciences Meeting and Exhibit, Reno, Nevada, January 11-14, 1993.

${ }^{3}$ McLachlan, B. and Bell, J., "Pressure-Sensitive Paint in Aerodynamic Testing," Experimental Thermal and Fluid Science, Vol. 10, No. 4, pp. 470-485.

${ }^{4}$ Torgerson, S., Liu, T., and Sullivan, J., "Use of Pressure Sensitive Paints in Low Speed Flows," AIAA Paper 96-2184, Advanced Measurement and Ground Testing Technology Conference, New Orleans, Louisiana, June 17-20, 1996.

${ }^{5}$ Coleman, P.B., Wallis, S.B., Dale, G.A., Jordan, J.D., Watkins, A.N., Goss, L., Davis, C.P.N., and Walter, T.M., “A Comparison of Pressure Sensitive Paint (PSP) Techniques for Aerodynamic Testing at Slow Velocities," Society of Automotive Engineers Paper 2002-01-255, SAE 2002 World Congress and Exhibition, Detroit, Michigan, March 2002.

${ }^{6}$ Jordan, J.D., Watkins, A.N., Davis, J., Weaver, W.L., Dale, G.A., Navarra, K., Urban, J., Devoid, W., and Stange, R., "Pressure-Sensitive Paint Measurements in a Large Scale Commercial Engine Test Stand," Proceedings of the 18 $8^{\text {th }}$ International Congress on Instrumentation in Aerospace Simulation Facilities, Toulouse, France, June 14-17, 1999.

${ }^{7}$ Carroll, B., Abbott, J. Lukas, E., and Morris, M., "Pressure Sensitive Paint Response to a Step Pressure Change," AIAA Paper 95-0483, $33^{\text {rd }}$ Aerospace Sciences Meeting and Exhibit, Reno, Nevada, January 9-12, 1995.

${ }^{8}$ Winslow, N., Carroll, B., and Setzer, F., "Frequency Response of Pressure Sensitive Paints," AIAA Paper 96-1967, 27 ${ }^{\text {th }}$ Fluid Dynamics Conference, New Orleans, Louisiana, June 17-20, 1996.

${ }^{9}$ Liu, T., Campbell, B., Burns, S., and Sullivan, J., "Temperature- and Pressure-Sensitive Luminescent Paints in Aerodynamics," Applied Mechanics Review, Vol. 50, No. 4, 1997, pp. 227-246.

${ }^{10}$ Bell, J.H., Schairer, E.T., Hand, L.A., and Mehta, R.D., "Surface Pressure measurements Using Luminescent Coatings," Annu. Rev. Fluid Mech., Vol. 33, 2001, pp. 155-206.

${ }^{11}$ Liu, T., and Sullivan, J.P., "Pressure and Temperature Sensitive Paints (Experimental Fluid Dynamics)," Springer-Verlag, Berlin, 2004

${ }^{12}$ Lackowicz, J., Principles of Fluorescence Spectroscopy, Second Edition, Plenum Press, New York, 1999, Chapter 8.
} 
${ }^{13}$ Asai, K. Kanda, H., and Kunimasu, T., "Detection of Boundary-Layer Transition in a Cryogenic Wind Tunnel by Using Luminescent Paint," AIAA Paper 96-2185, Advanced Measurement and Ground Testing Technology Conference, New Orleans, Louisiana, June 17-20, 1996.

${ }^{14}$ Asai, K., Kanda, H., Cunningham, C.T., Erausquin, R., and Sullivan, J.P., "Surface Pressure Measurement in a Cryogenic Wind Tunnel Using Luminescent Coating," Proceedings of the $17^{\text {th }}$ International Congress on Instrumentation in Aerospace Simulation Facilities, Pacific Grove, California, September 29-October 2, 1997, pp. 105-114.

${ }^{15}$ Upchurch, B.T. and Oglesby, D.M., "New PSP Developments at NASA Langley Research Center - Low Temperature PSP," Proceeding of the $6^{\text {th }}$ Annual Pressure Sensitive Paint Workshop, Seattle, Washington, October 6-8, 1998, pp. 10-1 - 1024.

${ }^{16}$ Asai, K., Amao, Y., Iijima, Y., Okura, I., and Nishide, H., "Novel Pressure-Sensitive Paint for Cryogenic and Unsteady Wind Tunnel Testing," AIAA Paper 2000-2527, $21^{\text {st }}$ AIAA Aerodynamic Measurement Technology and Ground Testing Conference, Denver, Colorado, June 19-22, 2000.

${ }^{17}$ Watkins, A.N., Goad, W.K., Obara, C.J., Sprinkle, D.R., Campbell, R.L., Carter, M.B., Pendergraft, O.C., Bell, J.H., Ingram, J.L., Oglesby, D.M., Underwood, P.J., and Humber, L.R., "Flow Visualization at Cryogenic Conditions Using a Modified Pressure Sensitive Paint Approach," AIAA Paper 2005-0456, 43 ${ }^{\text {rd }}$ Aerospace Sciences Meeting and Exhibit, Reno, Nevada, January 10-13, 2005.

${ }^{18}$ Germain, E. and Quest, J., "The Development and Application of Optical Measurement Techniques for High Reynolds Number testing in Cryogenic Environment," AIAA Paper 2005-0458, 43 ${ }^{\text {rd }}$ Aerospace Sciences Meeting and Exhibit, Reno, Nevada, January 10-13, 2005.

${ }^{19}$ Masuda, T., Isobe, E., and Higashimura, T., "Poly[1-(trimethylsilyl)-1-propyne]: A New Polymer Synthesized With Transition-Metal Catalysts and Characterized By Extremely High Gas Permeability," Journal of the American Chemical Society, Vol. 105, No. 25, 1983, pp. 7473-7474.

${ }^{20}$ Hamner, M.P., Popernack, Jr., T.G., Owens, L.R., and Wahls, R.A., "Using Temperature Sensitive Paint Technology," AIAA Paper 2002-0742, 40 ${ }^{\text {th }}$ AIAA Aerospace Sciences Meeting and Exhibit, Reno, Nevada, January 14-17, 2002.

${ }^{21}$ Amer, T.R., Obara, C.J., and Liu, T., "Quantifying the Effect of Pressure Sensitive Paint on Aerodynamic Data," AIAA Paper 2003-3951, $21^{\text {st }}$ Applied Aerodynamics Conference, Orlando, Florida, June 23-26, 2003. 